\title{
Multivariable $\mathrm{MHz}$ and $\mathrm{GHz}$ Wireless Chem/Bio Sensors for Environmental, Industrial, and Security Applications
}

\author{
Radislav A. Potyrailo ${ }^{1}$, Hacene Boudries ${ }^{2}$, and Rajesh R. Naik ${ }^{3}$ \\ ${ }^{\prime}$ GE Global Research Center, Niskayuna, NY, USA \\ ${ }^{2}$ Morpho Detection, Inc. Wilmington, MA, USA \\ ${ }^{3}$ Air Force Research Laboratory, Wright-Patterson AFB, OH, USA \\ Corresponding e-mail address: Potyrailo@crd.ge.co
}

\begin{abstract}
To improve selectivity of existing sensors and sensor arrays, we developed a concept of multivariable resonant sensing. Here we demonstrate its performance on several types of transducers in their wireless implementations. These transducers include inductor-capacitor-resistor (LCR), quartz crystal microbalance (QCM), and split ring resonator (SRR) devices. Our individual sensors demonstrate part-per-billion and part-per-trillion detection limits and successfully compete in their selectivity with sensor arrays.
\end{abstract}

Key words: selectivity, wireless implementations, inductor-capacitor-resistor (LCR), quartz crystal microbalance (QCM), split ring resonator (SRR)

\section{Introduction}

Chemical detection using existing sensors and their arrays is facing a major challenge of poor selectivity [1, 2]. Gas responses of sensors can be considered on several levels ranging from response to almost all gases, to response to only a specific gas, and finally to response to the gas of interest mixed with interferences and under uncontrolled operating conditions (Fig. 1). Most sensing materials respond not only to target analytes, but rather to a wide variety of gases (Fig. 1A). Gaseous interferences (Fig. 1B) significantly degrade sensor accuracy. The uncontrolled variable operating conditions (Fig. $1 \mathrm{C})$, further reduce sensor accuracy.

In wireless sensing, attachment of non-selective sensors to an analog input of a digital radio frequency identification (RFID) sensor does not improve selectivity [2]. Our approach for selective sensing involves materials with vapordependent response mechanisms coupled to a multivariable transducer.

Initially, we realized this approach using attractive passive RFID tags adapted for chemical sensing $[3,4]$. Here we illustrate performance of several types of wireless transducers such as QCMs [5, 6] and SRRs. Performance of developed sensors is illustrated in humidity-independent detection of organic vapors, detection of chemical agent simulants, and detection of explosives.
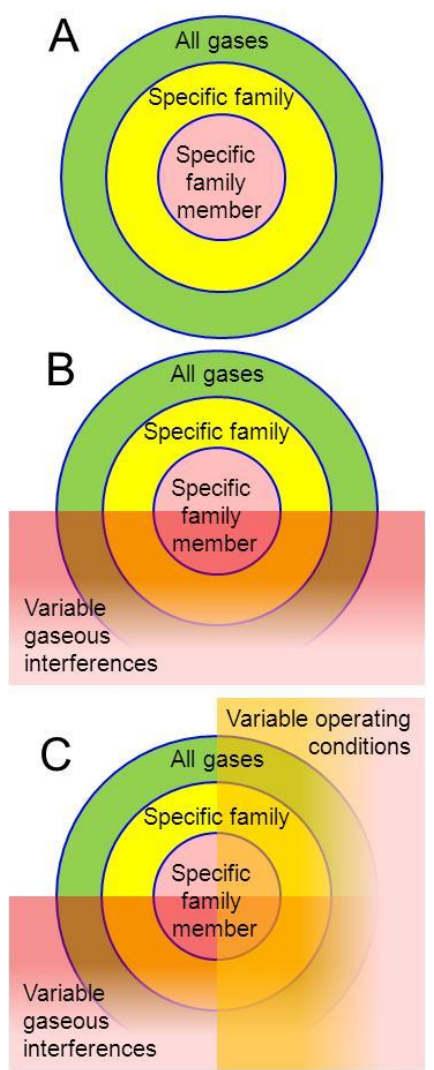

Fig. 1. Levels of needed performance of sensors: (A) Sensor nonselectively responds to any gas, or has partial selectivity to gases of a specific family, or has a high selectivity to particular gas from a specific family. Needed sensor operation in the presence of variable gaseous interferences (B) and under variable operating conditions $(C)$. 


\section{Experimental}

In this study, we utilized three types of multivariable wireless transducers (Fig. 2A-C) such as passive RFID devices (used here as LCR transducers), QCM, and SRR transducers. For selective sensing with individual sensors, we collected impedance spectra and their several derived parameters (Fig. 2D) and performed multivariate spectral analysis $[3,4]$ to quantify analytes. Our transducers operate in the $\mathrm{MHz}$ (RFID and QCM) and in the $\mathrm{GHz}$ (SRR) ranges. Measurements were performed with laboratory benchtop network analyzers.

\section{Results and Discussion}

It is important to evaluate capabilities of wireless devices with sensors attached to their analog input. This evaluation provides the insight on the opportunities and limitations of digital sensors for selective detection of vapors.

The operation principle of a typical digital RFID sensor with two sensing examples is shown in Fig. 3. This sensor contains an integrated circuit (IC) chip with an analog input from a transducer (Fig. 3A). Such IC chips often have a one-bit resolution for threshold measurements. Higher resolution (8-12 bit) is possible with an increase of IC chip cost. Such IC chips are available for operation at $125-135 \mathrm{kHz}$ (LF, low frequency), 13.56 MHz (HF, high frequency), and 868 - 956 $\mathrm{MHz}$ (UHF, ultrahigh frequency) ranges.

Operation of such LF and UHF RFID sensors with a conventional analog chemicapacitor attached to IC analog input is shown in Figs. 3B and $3 \mathrm{C}$. Similar to the operation of tethered individual transducers, individual wireless sensors did not discriminate between different types of vapors of similar dielectric constant.

For comparison of responses of a digital RFID sensor (Fig. 3B) and our multivariable RFID sensor (Fig. 4) we used three model vapors (water, toluene, and tetrahydrofuran) and a polyetherurethane sensing film [2]. Responses of the digital sensor to water and THF vapors differed only in magnitude, making impossible to differentiate between THF or water vapor, or to obtain selective responses to toluene or THF in the presence of water vapor.

However, a single passive RFID sensor with multivariable response discriminated between these three vapors. The individual responses (Fig. 4A-B) were analyzed using the principal components analysis (PCA) tools [7] as shown in Fig. 4C. Thus, a single multivariable response wireless sensor reliably discriminated between these three example vapors.
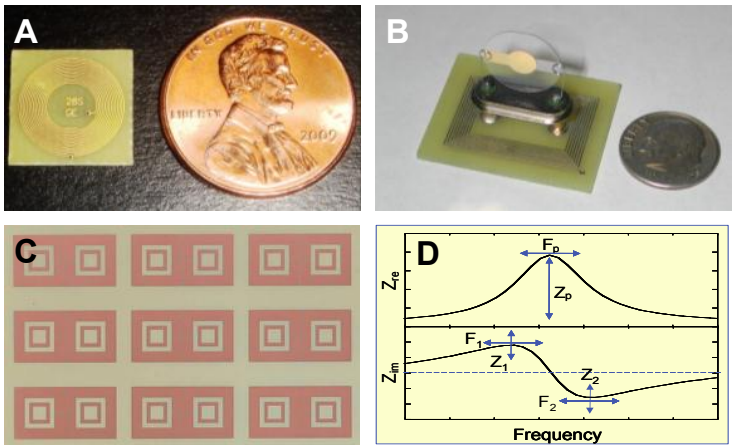

Fig. 2. Vapor detection in $\mathrm{MHz}$ and $\mathrm{GHz}$ spectral range: (A) RFID sensor, $M H z$ range; (B) $Q C M$ sensor, $\mathrm{MHz}$ range; (C) Array of dual-SRR sensors, $\mathrm{GHz}$ range; (D) Measured impedance spectrum (real part $Z_{r e}$ and imaginary part $Z_{i m}$ of impedance) and representative parameters for multivariate analysis: frequency $F p$ and magnitude $Z p$ of maximum of $Z_{r e}$, resonant $F 1$ and anti-resonant $F 2$ frequencies of $Z_{\text {im, }}$, and magnitudes $Z 1$ and $Z 2$ at resonant and antiresonant frequencies of $Z_{\text {im }}$.

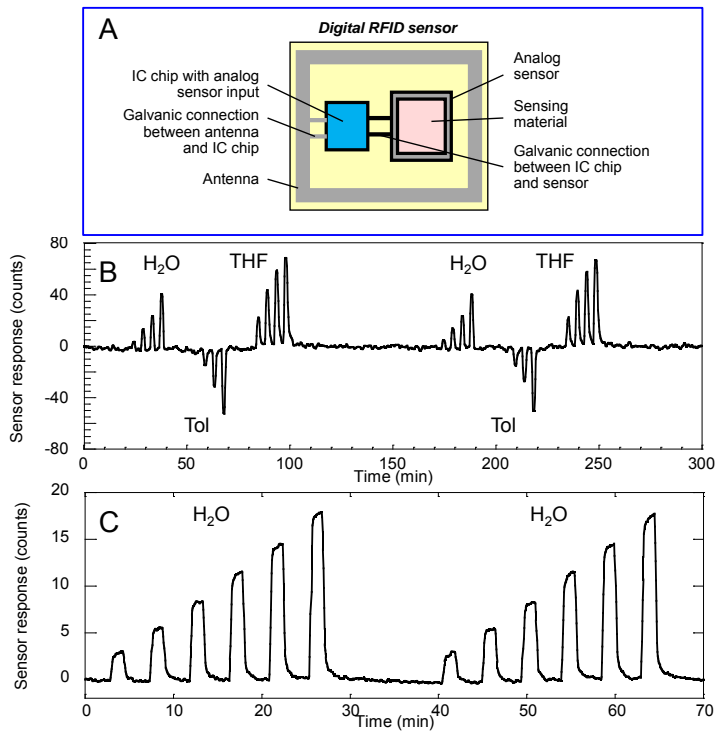

Fig. 3. Our experimental results on operation of digital RFID sensors with specialized IC chips with an analog input. (A) Schematic of a digital RFID sensor. (B) Detection of water (H2O), toluene (Tol), and tetrahydrofuran (THF) vapors with a chemicapacitor and a $125-\mathrm{kHz}$ digital RFID sensor. Concentrations of vapors: $0.18,0.36,0.53,0.71 P / P_{0}$. (C) Detection of water vapor with a chemicapacitor and a $915-\mathrm{MHz}$ digital RFID sensor. Concentrations of water vapor: $0.04,0.09,0.13,0.18,0.22,0.27 P / P_{0} . P_{0}$ is the saturated vapor pressure of a vapor under test and $P$ is the partial pressure of the vapor generated using our vapor generator.

Our multivariable response RFID sensors were further tested for their selective quantitation of analyte vapors in mixtures with two interferences [2]. Acetone was selected as a model analyte vapor to be quantitated in mixtures with water and ethanol vapors. Fig. $5 \mathrm{~A}$ illustrates the PCA scores plot of sensor 
response to variable concentrations of acetone vapor mixed with water and ethanol vapors. A correction of acetone response at different levels of water and ethanol vapors was done using PCA. The resulting multivariate calibration curves for acetone were not affected by the variable levels of water and ethanol vapors (Fig. 5B) and provided a new capability to quantify an analyte vapor in the presence of multiple interferences using only one sensor.

Detection of several explosives and oxidizers was demonstrated using RFID sensors. In these initial experiments, the main emphasis was to evaluate the detection capability of the sensors. Fig. 6 illustrates detection of $0.2 \mathrm{mg}$ of trinitrotoluene (TNT), $0.1 \mathrm{mg}$ of triacetone triperoxide (TATP) in acetonitrile, and $5 \mathrm{~g}$ of $\mathrm{NH}_{4} \mathrm{NO}_{3}$ using developed RFID sensors. Detailed sensitivity and selectivity of the RFID sensors will be evaluated in future studies.

An example of a QCM sensor response with the wireless signal collection is shown in Fig. 7. In this experiment, the polymer-coated QCM was evaluated for its response to $\mathrm{CO}_{2}$ as a model gas of interest for industrial monitoring [5]. In the experiments for the evaluation of response hysteresis in dry air, the sensor provided a hysteresis-free response.
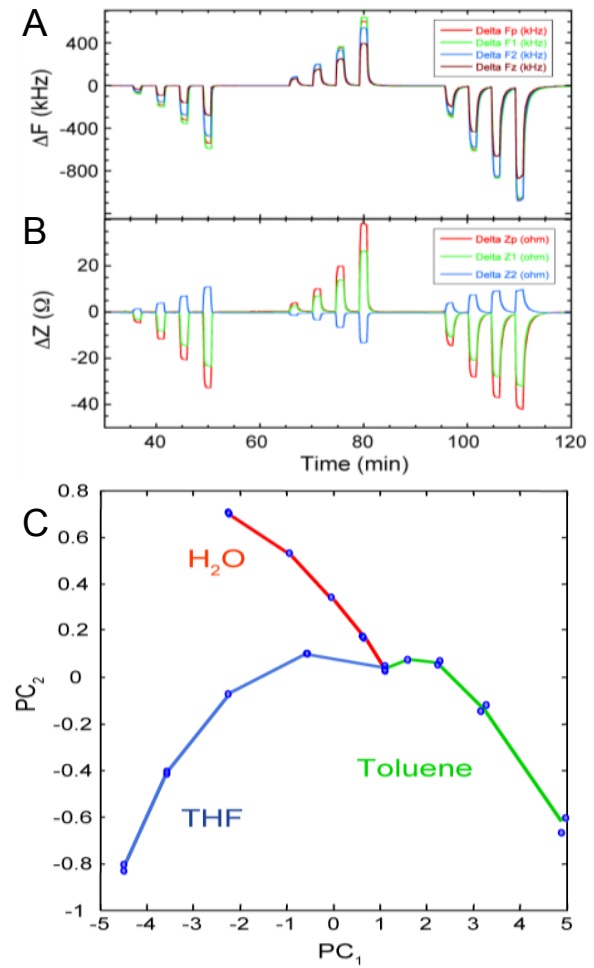

Fig. 4. Ability of developed multivariable RFID sensors to discriminate between three individual model vapors (water, toluene, and THF): Individual sensor responses (A) Fp, F1, F2, FZ and (B) Zp, Z1, Z2. (C) Scores plot of a PCA model. Vapor concentrations: $0.18,0.36,0.53$, and $0.71 P / P_{0}$.
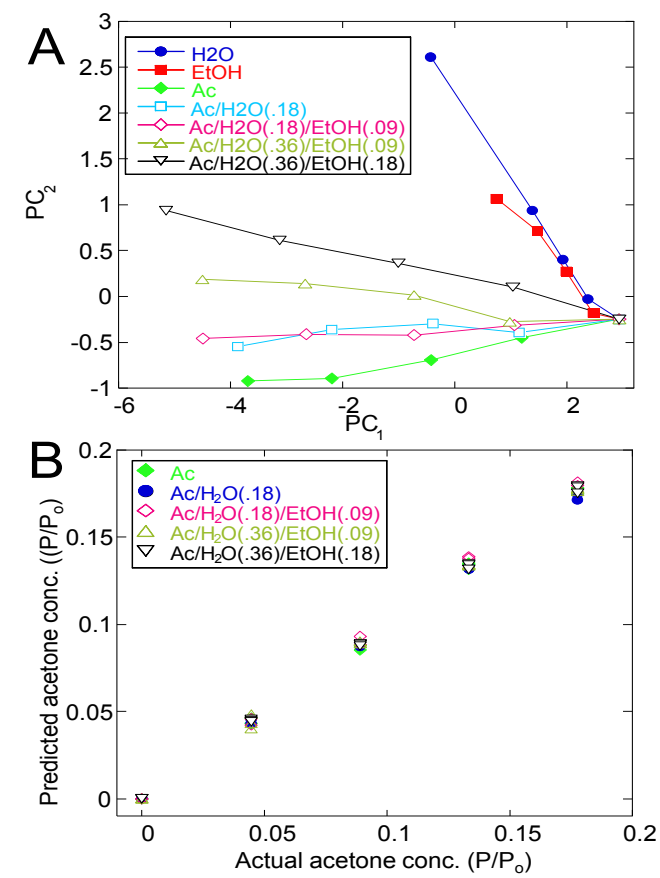

Fig. 5. Quantitation of model analyte (acetone) in the presence of two interferences (water and ethanol vapors): (A) Plot of PC1 vs PC2 illustrates sensor response to four concentrations of acetone $(0.044$, $\left.0.089,0.133,0.178 \mathrm{P} / \mathrm{P}_{0}\right)$ at two concentrations of water $\left(0.18,0.36 P / P_{0}\right)$ and two concentrations of ethanol $\left(0.09,0.18 P / P_{0}\right)$. (B) Multivariate calibration curves for acetone mixed with interferences.
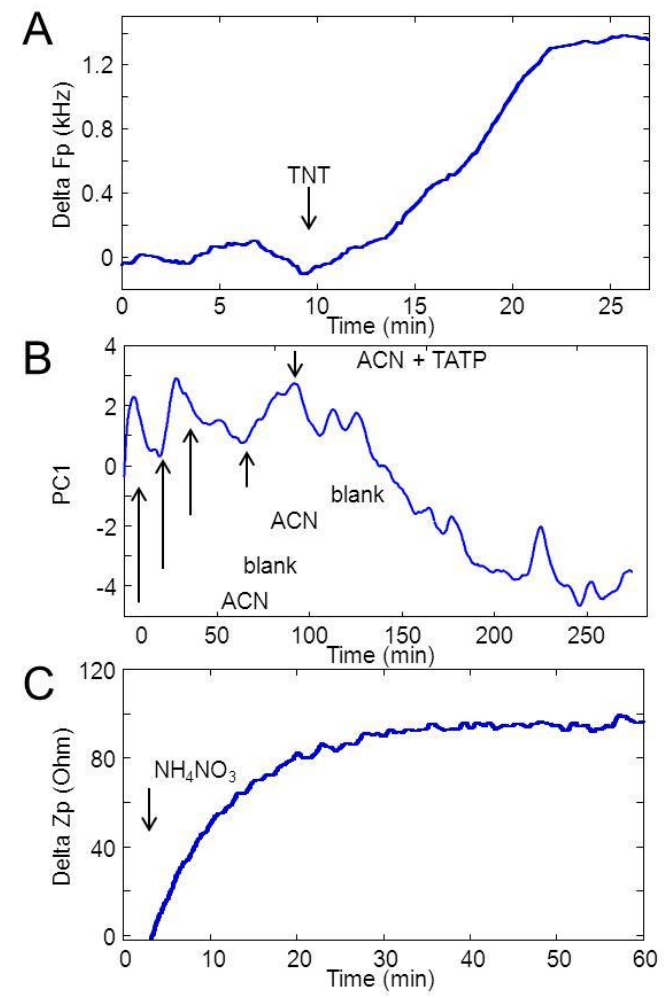

Fig. 6. Examples of detection of (A) $0.2 \mathrm{mg}$ of TNT (powder form), (B) $0.1 \mathrm{mg}$ of TATP in dissolved in acetonitrile $(A C N)$, and (C) $5 \mathrm{~g}$ of $\mathrm{NH}_{4} \mathrm{NO}_{3}$ (crystal form) using developed sensors. 


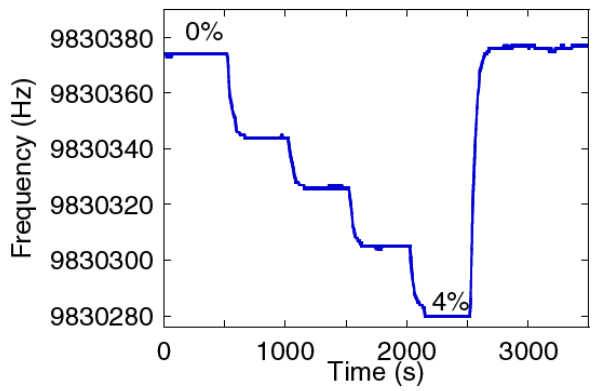

Fig. 7. Response of our developed wireless QCM sensor to $\mathrm{CO}_{2}$ gas introduced into the environmental chamber in steps of 1, 2, 3, and $4 \%$.

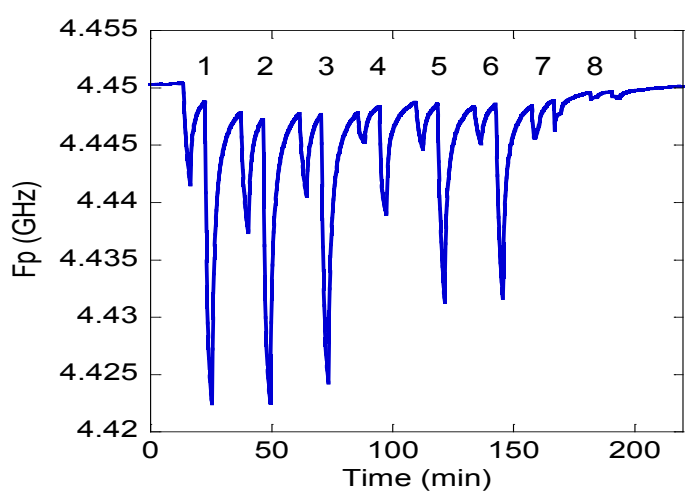

Fig. 8. Initial results of vapor sensing with a dualSRR transducer coated with an $A u-A 3$ peptide sensing film. Eight vapors (1-8) were at $P / P_{0}=0.044$ and 0.089. Water background was at $P / P_{0}=0.18$. Vapors: (1) acetonitrile, (2) dichloromethane, (3) methyl salicylate, (4) ethanol, (5) toluene, (6) 1pentanol, (7) chloroform, (8) salicylaldehyde.

We also evaluated performance of the $\mathrm{GHz}$ sensors based on the dual-SRR structures. In these experiments, we utilized peptide-capped Au nanoparticles (Au-A3) [8] as a sensing film and tested the response of these $\mathrm{GHz}$ sensors to eight vapors (Fig. 8).

\section{Conclusions}

Operation of resonant structures of different designs at $\mathrm{MHz}-\mathrm{GHz}$ frequencies provides several opportunities when the multivariable signal acquisition and analysis are performed. First, different tranducers provide diverse opportunities to probe vapor responses of sensing materials. Second, different operating frequencies bring additional possibilities related to the material dispersion of different sensing materials and detected analytes.

Besides the transducer types described here (LCR, QCM, SRR), there are other transducers that should benefit from their wireless excitation and the multivariable signal acquisition and analysis.

\section{Acknowledgements}

The authors would like to acknowledge E. Downey, W. Morris, Y. Lee, N. Nagraj, C. Surman, J. M. Slocik, and H. Lai for technical assistance and discussions. This work has been supported in part by GE Corporate fundamental research funds, by National Institute of Environmental Health Sciences (Grant No. 1R01ES016569-01A1) and by Department of The Air Force, Air Force Research Laboratory (Contract FA8650-08-C6869).

\section{References}

[1] F. Röck, N. Barsan and U. Weimar, Electronic Nose: Current Status and Future Trends, Chem. Rev., 108, 705-725 (2008); doi: 10.1021/cr068121q

[2] R. A. Potyrailo, C. Surman, N. N. Nagraj and A. Burns, Materials and Transducers Toward Selective Wireless Gas Sensing, Chem. Rev., 111, 7315-7354 (2011); doi: $10.1021 / \mathrm{cr} 2000477$

[3] R. A. Potyrailo and W. G. Morris, Multianalyte Chemical Identification and Quantitation Using a Single Radio Frequency Identification Sensor, Anal. Chem., 79, 45-51 (2007); doi: 10.1021/ac061748o.

[4] R. A. Potyrailo, C. Surman, S. Go, Y. Lee, T. Sivavec and W. G. Morris, Development of radio-frequency identification sensors based on organic electronic sensing materials for selective detection of toxic vapors, J. Appl. Phys., 106, 124902 (2009); doi: 10.1063/1.3247069.

[5] W. G. Morris and R. A. Potyrailo, Wireless Sensor Array System for Combinatorial Screening of Sensor Materials, MRS Symp. Proc., 894, 219-224 (2006); doi: 10.1557/PROC0894-LL07-02.

[6] R. A. Potyrailo and W. G. Morris, Wireless resonant sensor array for high-throughput screening of materials, Rev. Sci. Instrum., 78, 072214 (2007); doi: 10.1063/1.2755657.

[7] B. A. Snopok and I. V. Kruglenko, Multisensor systems for chemical analysis: state-of-the-art in Electronic Nose technology and new trends in machine olfaction, Thin Solid Films, 418, 21-41 (2002); doi: 10.1016/S0040-6090(02)00581-3.

[8] J. M. Slocik, M. O. Stone and R. R. Naik, Synthesis of gold nanoparticles using multifunctional peptides, Small, 1, 1048-1052 (2005); doi: 10.1002/smll.200500172. 